folding fan when illuminated.

Yanke Che and his

colleagues at the Beijing

National Laboratory for

Molecular Sciences created

thin, ribbon-like structures up

to one micrometre wide.

The ribbons are composed of multiple layers, each consisting of pairs of a long, thin molecule called perylene diimide. Under a blue-green laser, the layers slide apart because the photons excite electrons and distort molecular conformations, the researchers say. As a result, the ribbons expand, reaching around 12 micrometres in width after 3 minutes. They shrink back in seconds when exposed to an electron beam.

Materials that change shape under light could have many applications, including in artificial muscle, the team says. Adv. Mater. http://doi.org/f2v7vc (2014)

\section{CONSERVATION GENETICS}

\section{Leopard-skin origins traced}

DNA analysis can reveal the origins of products from endangered species, which could help to curb illegal trade.

Such goods are often seized far from their origins, making it hard to know where to focus enforcement. Samrat Mondol of the National Centre for Biological Sciences in Bangalore, India, and his colleagues designed a DNA test that enabled them to trace the geographic origins of 40 seized leopard pelts (from Panthera pardus; pictured) to within a few hundred kilometres. They compared DNA from the pelts to that from blood and faecal samples taken from 173 leopards, focusing on gene variants found in certain locations in India. Very few of the skins were local to their seizure point. Central India appears to be a leopard

poaching hotspot.

The technique could easily

be used for other traded

species, the authors say.

Conserv. Biol. http://doi.org/w5s (2014)

\section{MICROBIOLOGY}

\section{Beware tainted microbe studies}

DNA contamination is ubiquitous in laboratory reagents commonly used to analyse the microbes that inhabit the human body.

Susannah Salter at the Wellcome Trust Sanger Institute in Hinxton, UK, Alan Walker at the University of Aberdeen, UK, and their colleagues used off-the-shelf DNA-extraction kits and two common techniques to sequence a pure culture of the bacterium Salmonella bongori as well as a series of diluted versions. Contamination by other bacterial species increased with each dilution, and quickly drowned out the original S. bongori signal. The team traced at least part of the problem to the DNAextraction kits, which are not sold as sterile.

This contamination could undermine microbiome studies, especially in samples that have low microbial content, including those from spinal fluid, blood and the lungs, the authors say. BMC Biol. 12, 87 (2014)

\section{ASTRONOMY}

\section{Merged stars dodge black hole}

A mysterious cloud-like object that survived a close encounter with a black hole might be a merged pair of stars.

Andrea Ghez of the

University of California in Los Angeles and her team used the Keck telescopes on Mauna Kea in Hawaii to observe the object, called G2. In March, it was nearly engulfed by our Galaxy's central supermassive black hole.

Previous observations using specific wavelengths

SOCIAL SELECTION Romitariater

\title{
Unusual reference attracts notoriety
}

An editorial oversight has turned a report on fish pigmentation into one of the year's most talked-about papers. The study of poeciliid fishes, first published online in July by the journal Ethology, received scant attention until ecologist David Harris at the University of California, Davis, tweeted a screenshot of one of its pages, highlighting this phrase in parentheses: "Should we cite the crappy Gabor paper here?" Harris added his own comment on Twitter: "Not sure how this made it through proofreading, peer review and copy editing." In one of dozens of responses, Tim Elfenbein, managing editor of the journal Cultural Anthropology, tweeted: "Note to authors: you are ultimately responsible for the work that bears your name, no matter the level of editing." Ethology 120, 1090-1100 (2014)

\section{( $)$ Based on data from altmetric.com Altmetric is supported by Macmillan Science and Education, which owns Nature Publishing Group.}

DNATURE.COM

For more on popular papers: go.nature.com/3bswdt of light indicated that it was a young cloud of gas, which would have been stretched or devoured by the black hole. But the team's infrared images showed no clear change in G2's appearance. Instead, the researchers suggest that the object is a pair of stars that have recently merged, perhaps owing to the presence of the black hole.

The black hole's gravity could be disrupting the dynamics of nearby binary systems, causing them to coalesce, according to the authors.

Astrophys. J. Lett. 796, L8 (2014)

\section{CLIMATE SCIENCE}

\section{Water vapour predicts flooding}

Streams of concentrated water vapour in the atmosphere could be used to predict flooding in Europe more accurately than rainfall does.

A team led by David Lavers of the European Centre for Medium-Range Weather Forecasts in Reading, UK, looked at forecasts from last winter, when the United Kingdom and other parts of Europe saw major flooding

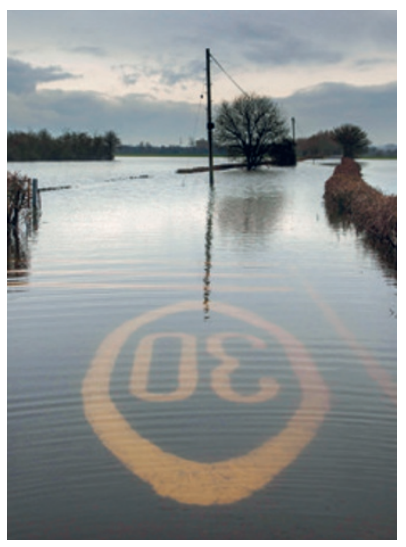

(pictured). By incorporating information on the transport of water vapour in the atmosphere, the team found that scientists could have predicted flooding in some areas of Europe by up to three extra days.

The weather patterns associated with these atmospheric rivers do not break apart as rapidly as rainfall-related patterns do, making them more reliable flood predictors, the team says. Nature Commun. 5, 5382 (2014)

\section{DNATURE.COM}

For the latest research published by Naturevisit:

www.nature.com/latestresearch 